\title{
Quo vadis quantitative sustainability assessment?-a personal statement
}

\author{
Subhas Sikdar ${ }^{1}$
}

Accepted: 4 August 2021 / Published online: 21 August 2021

(c) The Author(s), under exclusive licence to Springer-Verlag GmbH Germany, part of Springer Nature 2021

More than two decades have passed since I started promoting the technical need for measuring sustainability of technologies. My activities spanned a wide spectrum comprising founding the Sustainable Engineering Forum (SEF) of the American Institute of Chemical Engineering (AIChE) and organizing technical sessions in its annual meetings, organizing specialty conferences under the Engineering Foundation (now called Engineering Conferences International), creating a NATO Pilot project called Clean Products and Processes with member delegates from 25 countries, and organizing annual meetings for ten years in various cities in Europe, and writing and editing several books and articles specifically on measurement of sustainability (for example, Sikdar 2019; Sikdar et al. 2017). Two decades later, when I look back to take a stock of what advancement has been made on the topic, disappointment is hard to hide. The symptoms of the problem are several-fold: first, most authors of technical papers either declare a process or product as sustainable with no validation, second, others only focus on potential reduction of greenhouse gases and conclude that the research outcomes denote attainment of sustainability, and still others adopt highly technical process integration featuring just a few technical variables that can help or hurt with sustainability goals and claim that the optimized process or product as sustainable. More on this engineering approaches later.

The problem of green methods of process, product, or operation is quite similar. Announcing greenness seems to be sufficient, even when it is transparently self-serving. One reason for this failure is that most papers dealing with sustainability or greenness come from academia, and academic studied are funded by government agencies. These agencies

Subhas Sikdar: Editor in Chief.

Subhas Sikdar

subhas.sikdar@gmail.com

1 Cincinnati, USA fund specific projects focused on narrowly described topics that are amenable to scientific investigation by individual investigators. There is never enough funding that would be required for comprehensive research. The arrangement is also not suitable for the need of a graduate student desirous to soon obtain a degree to join the workforce. These and other factors create a mismatch between what is needed and what can be offered. I do not view this deficiency as a willful act but as resulting from the limitation of the research conditions.

The essential ingredients needed for sustainability assertion are the realization that (1) sustainability is about a system that needs to be clearly defined, (2) scale of the system, which can be a technology, or a corporation with multiple technologies, a complex ecosystem, or finally a regional or national level system, and (3) it is imperative to identify the specific metrics or indicators that need to be evaluated for sustainability determination quantitatively. The ensemble of indicators used for a problem at one scale is also different from that at a different scale. At the technology scale, most of the indicators can be technical, and as one moves along the scale toward a country level study, policy indicators dominate. It is important to realize that in a world with constant changes, sustainability is not an absolute concept, but necessarily a relative or comparative one. It also changes with time, as conditions change. Also, sustainability is environmental, economic as well as sociological. A bottom-up approach to sustainability determination is based on the idea that technology systems, when done properly, will aggregate into a larger scale technical sustainability. This approach is natural for scientific and engineering research, but there is no proof that the individual small-scale sustainability will integrate to a large-scale sustainability. On the other side of the technology-policy spectrum, one could start with a policy prescription for a region or a country, and technology development will accommodate the policy suggestions. This approach emanates from the political control of the research 
apparatus and is vulnerable to missteps leading to wasting of resources and less than useful outcomes.

Most countries have developed their own programs for attaining sustainability. Typically, there will be a litany of indicators with quantitative aspirational goals. At the regional levels, the indicators may not all be quantitative. Though there are some suggested methods for determining the values of some indicators, the individual countries determine how they would measure and report them. The results are reported as a time series data of how the individual indicators are performing over time. Whether or not a particular country or a region is making real progress towards sustainability can be assessed by looking up the time series data. But it is highly likely that not all indicators will tend toward a better future, making overall assessment difficult and subjective. The idea of relative sustainability is embedded in the time series data.

For the technology systems, one may have several alternatives to choose from, and the indicator data can be used to make the choice. However, aggregating the indicator values into one score is the easiest way to make that determination. Several aggregation schemes can be used but they need to be transparent and verifiable by other researchers. They also need to be scientifically sound. Although some industrial product or process systems have been treated this way, this approach has not gained much traction either in industry or in academia. For process systems, the technique of simulation is used for carrying out process optimization for one variable, such as energy minimization, or multiple variables, such as simultaneous energy and water use minimization. The methods are sound so long as they treat realistic systems. The problem is that a comprehensive approach that takes into consideration all possible stressor data is missing in these computer-aided approaches. An alternate approach is to collect indicator data as comprehensibly as possible to represent sustainability conditions and evaluate the process data compared to a benchmark. Typically, this is done using a rational life cycle assessment framework for which the relevant data are available. The limitation of this data-based approach is that the conditions before and after are fixed, and no other combinations of process data can be predicted for their eventual comparative state vis a vis the benchmark. In other words, the assessment is retrospective and prospective. One would think that a simulation approach is inherently amenable to treat situations where the values of the indicators are changeable. But this has not been achieved yet. The predictive ability of simulation approaches is limited except for minor excursions beyond the area of study.
Once a technology is fixed for real-world application, it can rarely be changed in the short term. But in the technology climate of rapid changes, the economic risks are asymmetric to profit gain. We must be able to make reliable prediction for a simulation-based technology worthy of an investor's money. The investor has a skin in the game and is unlikely fall for uncertainty. If we are looking for innovation for significant improvement, these techniques are thus woefully insufficient. Therefore, the approach of process synthesis where novel processed are created in-silico will find resistance to acceptance. Innovations require real advances in chemistry or biological sciences. There are some instances of significant improvement resulting from mathematical or graphical techniques, such as heat or mass integration, or combined heat and mass integration. However, sustainability systems are complex systems which are inherently resistant to fruitful prediction. More rigorous methods than multi-objective optimization techniques, used currently, are needed for optimizing a complex sustainability system.

From this vantage point of an editor, I have noticed a trend away from attempts on sustainability assessment that consider a meaningful litany of environmental, human health and ecosystem stressors, even using the minimum required for a standard life cycle assessment, toward attempts on assertions of sustainability based only on greenhouse gas reduction. This is lamentable, and it is wrong. The concern for air, water and land pollution with their toxicity impacts still exist, and they cannot be ignored in preference to greenhouse gas emission. Sustainability, as we understand, is concerned about amelioration of the adverse impacts on society, human health, and the environment. It is imperative that in sustainability assessment, or in assertions, we do not forget the overarching picture.

\section{References}

Sikdar SK (2019) Fractured state of decisions on sustainability: an assessment. Sustain Prod Consum 19:237

Sikdar SK, Sengupta D, Mukherjee R (2017) Measuring progress towards sustainability: a treatise for engineers. Springer International Publishing, Switzerland

Publisher's Note Springer Nature remains neutral with regard to jurisdictional claims in published maps and institutional affiliations. 